\title{
ROSAT/Chandra observations of a bright transient in M 81
}

\author{
K. K. Ghosh ${ }^{1, \star}$, D. A. Swartz ${ }^{2}$, A. F. Tennant ${ }^{3}$, and K. Wu ${ }^{4, \star \star}$ \\ 1 NRC, NASA Marshall Space Flight Center, SD-50, Huntsville, AL 35812, USA \\ 2 USRA, NASA Marshall Space Flight Center, SD-50, Huntsville, AL 35812, USA \\ e-mail: doug.swartz@msfc.nasa.gov \\ 3 Space Science Department, NASA Marshall Space Flight Center, SD-50, Huntsville, AL 35812, USA \\ e-mail: allyn.tennant@msfc.nasa.gov \\ 4 Mullard Space Science Laboratory, University College London, Holmbury St Mary, Surrey RH5 6NT, UK \\ and School of Physics A28, University of Sydney, NSW 2006, Australia \\ e-mail: kw@mssl.ucl.ac.uk
}

Received 19 July 2001 / Accepted 4 October 2001

\begin{abstract}
We present a 10-year X-ray light curve and the spectra of a peculiar X-ray transient in the spiral galaxy M 81. The source was below the detection limit of ROSAT PSPC before 1993, but it brightened substantially in 1993, with luminosities exceeding the Eddington limit of a 1.5- $M_{\odot}$ compact accretor. It then faded and was not firmly detected in the ROSAT HRI and PSPC observations after 1994. The Chandra image obtained in 2000 May, however, shows an X-ray source at its position within the instrumental uncertainties. The Chandra source is coincident with a star-like object in the Digitized-Sky-Survey. A Hubble image suggests that the optical object may be extended. While these three observations could be of the same object, which may be an X-ray binary containing a black-hole candidate, the possibility that the ROSAT and Chandra sources are two different objects in a dense stellar environment cannot be ruled out. The Hubble data suggests that the optical object may be a globular cluster yet to be identified.
\end{abstract}

Key words. X-rays: binaries - X-rays: galaxies - black hole physics - stars: binaries: close globular clusters: general

\section{Introduction}

M 81 (NGC 3031) is a nearby Sab spiral galaxy just beyond the Local Group. It has a well defined central bulge; its two spiral arms are clearly marked by strings of bright young stars.

M 81 was observed by Einstein in 1979 and by ROSAT over the period 1991-1998. The Einstein observations identified 10 point sources (including the nucleus) in the field (Fabbiano 1988). There are $46 \mathrm{HRI}$ and $69 \mathrm{PSPC}$ sources found in the deep images obtained by ROSAT (Immler \& Wang 2001). The Chandra observation was carried out in 2000. A total of 97 sources were detected in the ACIS S3 image (Tennant et al. 2001), which has an area of $8.3 \times 8$ ' 3 centered at the nuclear region. More sources are in the other S-chip images further away from the nucleus (Swartz et al. in preparation).

Send offprint requests to: K. K. Ghosh, e-mail: kajal.ghosh@msfc.nasa.gov

* NRC Senior Research Associate.

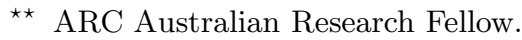

While a portion of the sources in the M 81 field are foreground stars and background AGNs, the majority are believed to belong to the galaxy or its dwarf satellites (Tennant et al. 2001; Immler \& Wang 2001). These X-ray sources form an inhomogeneous group, consisting of objects from canonical sources such as supernova remnants and X-ray binaries to exotic systems such as the very-soft sources discussed in Swartz et al. (2001). Many sources are variable, and some are transients showing dramatic brightening for a brief duration ( $\$ 1$ yr) (see Immler \& Wang 2001; La Parola et al. 2001).

X-ray transients are also commonly found in our Galaxy. Among the brightest are candidate blackhole X-ray binaries, whose peak luminosities may reach $10^{39}-10^{40} \mathrm{erg} \mathrm{s}^{-1}$ during the outbursts, and certain neutron-star X-ray binaries (including the type I bursters), which may be as bright as or even brighter than $2 \times 10^{38} \mathrm{erg} \mathrm{s}^{-1}$, the Eddington limit of a $1.5-M_{\odot}$ compact accretor. These luminous X-ray binaries can be detected easily everywhere within the Galaxy when they are in active states. (For a review of the properties of galactic X-ray 

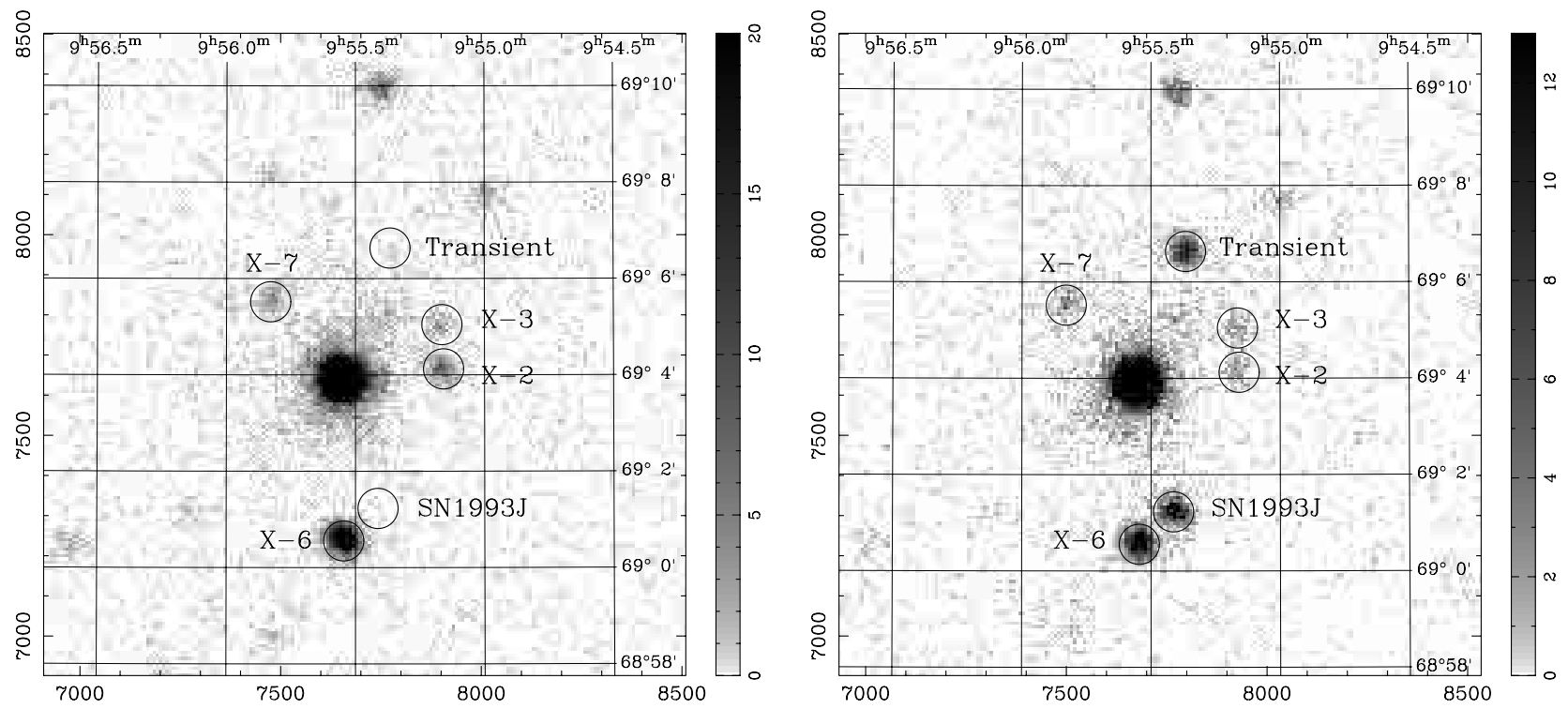

Fig. 1. Comparison of two ROSAT PSPC observations in 1992 and 1993 (left and right panels respectively). The bright nucleus is seen centered in the two images as well as several other sources in the field. One can see the brightening of the transient source P33 and the supernova SN 1993J. The color table has be adjusted by the integration time so that sources of equal flux levels appear at similar gray levels.

binaries, see articles in Lewin et al. 1995.) Transient X-ray binaries are found in globular clusters as well as in the Galactic bulge and disk. No candidate black hole X-ray binaries have been identified in Galactic globular clusters. The bright globular cluster sources in our Galaxy tend to be type I X-ray bursters (Verbunt \& van den Heuvel 1995). The most luminous globular cluster X-ray source in the Galaxy has a luminosity $\sim 5 \times 10^{37} \mathrm{ergs}^{-1}$ (Bloser et al. 2000).

Here, we report the analysis of the photometric and spectroscopic data of the peculiar transient source in the NW region of M 81 observed by ROSAT and Chandra. The nature of the source and its implications are discussed.

\section{Observations and data analysis}

The M 81 field was observed many times by ROSAT in the 1990s (Immler \& Wang 2001) including 9 PSPC observations spanning 1991 March to 1994 April and $11 \mathrm{HRI}$ observations (from 1992 Oct. to 1998 April). We examined these data using a combination of FTOOLS and LEXTRCT, a locally supported software package. ROSAT PSPC images obtained in 1992 and in 1993 are shown in the left and right panels of Fig. 1, respectively. The transient as well as SN 1993J are both seen in the 1993 data. In the 1992 data, there is only a very slight excess at the transient's position that is significant at the $2.4 \sigma$ level. To derive an accurate position, we adjusted the coordinates in each ROSAT image to align the nucleus to be at the radio position $9^{\mathrm{h}} 55^{\mathrm{m}} 33.17^{\mathrm{s}},+690355.1$ (Ma et al. 1998). This adjustment was typically $\sim 5$ arcsec. No rotation was applied. With this offset, the locations of SN 1993J and the bright Einstein sources X-2, X3 and X-6 are all consistent with their Chandra positions.
The transient is listed as source P33 in Immler \& Wang (2001) at $\left(9^{\mathrm{h}} 55^{\mathrm{m}} 22.7^{\mathrm{s}},+690631\right)$. In our adjusted coordinates, its position is $\left(9^{\mathrm{h}} 55^{\mathrm{m}} 21.96^{\mathrm{s}},+690638.0\right)$ with an uncertainty of 3.5 arcsec.

The M 81 field was observed for $50 \mathrm{ks}$ by Chandra on 2000 May 7 with the nucleus centered on the ACIS S3 chip. The Chandra image, data-analysis, and general properties of the sources found in the S3 image were presented in Tennant et al. (2001). The transient is less than 1 arcsec from a Chandra source at a position of $\left(9^{\mathrm{h}} 55^{\mathrm{m}} 21.95^{\mathrm{s}},+690637.8\right)$. The source is off-axis in the Chandra data, appearing roughly elliptical in shape, with Gaussian widths $\sigma=2$ and 1 arcsec in the two dimensions on the sky.

The Chandra position is less than 1 arcsec from the object listed as ID 50777 by Perelmuter \& Racine (1995) who report its brightness and colours are $V=16.87$, $B-V=0.97$, and $V-R=0.56$.

The region around object ID 50777 was observed with Hubble WFPC2 on 1998 April 17. The three images taken with the F450W filter, which roughly correspond to the $V$ band, were combined using a median filter to remove cosmic ray tracks. The final image was visually inspected and in particular object ID 50777 was compared to object ID 50658; another bright star-like object with $V=15.91$ (Perelmuter \& Racine 1995). Object ID 50658 shows diffraction spikes and is saturated in the core with indication of some charge leakage during read out. Object ID 50777 does not show diffraction spikes, nor does it appear to be saturated in the core. The background around both objects is partially resolved into stars and no attempt was made to remove these very faint stellar images.

The Tiny Tim software package (Krist \& Hook 1999) was used to compute model Point Spread Functions 


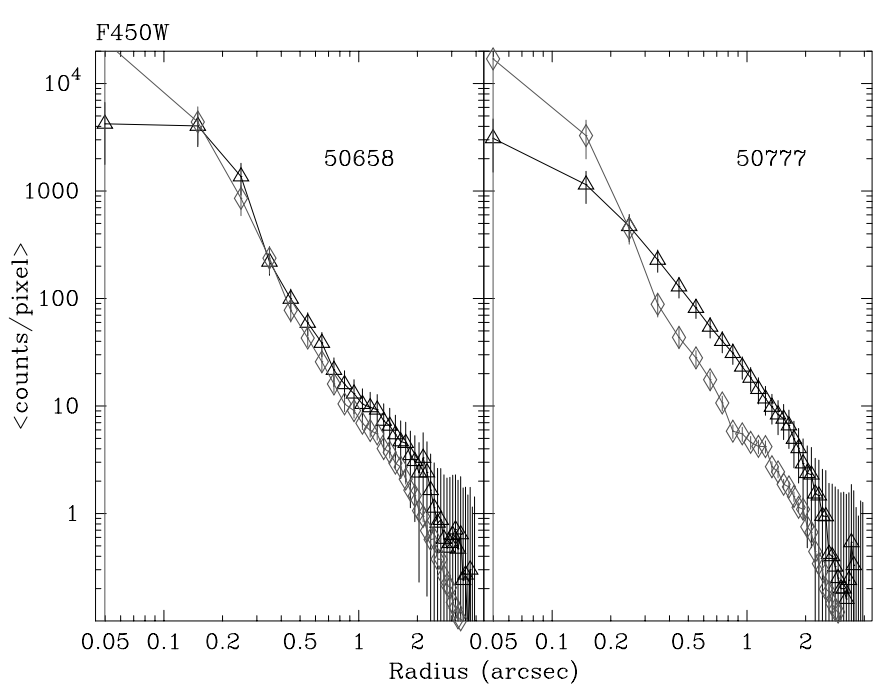

Fig. 2. The radial profile of two objects in the Hubble WFPC2 data with the F450W filter. The profile from the data are shown in black triangles. Tiny Tim model PSFs normalized to contain the same number of counts are shown with gray diamonds. The error bars are based on the scatter and thus represent the azimuth variation in the PSF as well as the variations in the background due to faint stars.

(PSFs) for the two locations. The radial profiles of the two sources as well as the profiles derived from the Tiny Tim model PSFs are shown in Fig. 2. To normalize the data we subtracted the average level in the outer three annuli(from roughly 3.7 to 4.0 arcsec). To normalize the Tiny Tim models we multiplied by the total number of counts detected in the data. As can be seen the PSF of ID 50658 is saturated in the core with an excess at 0.25 arcsec where the charge is finally read out. At larger radii, the model PSF approximates the data fairly accurately. The model PSF poorly approximates the data for object ID 50777. The radial profile of ID 50777 is very smooth and does not show the central core nor the excess at 1-2 arcsec (mainly due to the diffraction spikes). Thus, ID 50777 appears extended in the Hubble data. Not shown in the figure is the PSF for object ID 50696 (Perelmuter \& Racine 1995) which is also in the same Hubble field and was confirmed by Perelmuter et al. (1995) as a globular cluster in M 81. The radial profile of ID 50696 shows an extension very similar to that of object ID 50777 .

We conclude that object ID 50777 is extended in the Hubble data and is very likely a bright globular cluster in M 81. The half light radius in the Hubble data is 2.4 pixels. At the distance of $\mathrm{M} 81$ this corresponds to $4.4 \mathrm{pc}$. If we remove the estimated Hubble PSF then the half light radius would be about $3 \mathrm{pc}$ which is a fairly typical size for globulars in our galaxy (e.g., Ashman \& Zepf 1998).

\section{Brightness variation and spectroscopic properties}

The 10-year X-ray light curve of the transient source is shown in Fig. 3. The source was below the ROSAT HRI equivalent count rate of $\sim 3 \times 10^{-4} \operatorname{cts~s}^{-1}$ up to 1993 May in both the PSPC and HRI images. Its count rate, however, reached about $1.5 \times 10^{-2}$ cts $^{-1}$ during the flare (brightening) around 1993 September-November. It then faded within a 5 -month period and was marginally detected $(<2.5 \sigma)$ in the subsequent ROSAT observations at the brightness level of $\sim(2-3) \times 10^{-4}$ cts s $^{-1}$.

The equivalent ROSAT HRI count rate of the Chandra source is $(2.6 \pm 0.2) \times 10^{-4} \mathrm{cts} \mathrm{s}^{-1}$, consistent with the "quiescent" brightness of the source P33 seen in the ROSAT observations.

We extracted the ROSAT PSPC spectra of the source during the brightening in 1993 and grouped them into spectral bins containing a minimum of 20 counts. The spectra are fitted using XSPEC (Arnaud 1996) to simple functions. The best fitting parameter values as well as the 90 percent confidence single parameter uncertainties are shown in Table 1 . In all cases, the absorption column density is consistent with the line-of-sight Galactic value $\left(n_{\mathrm{H}}=4 \times 10^{20} \mathrm{~cm}^{-2}\right.$, Stark et al. 2001). The power-law and bremsstrahlung models provide equally acceptable fits. For the black-body model, the best fitting absorption column density is consistent with zero, and therefore, we fixed its value to the Galactic value. Based on the value of $\chi^{2}$ we cannot rule out a black-body model although it does not fit as well. When we add the two ROSAT data sets, the black-body model is still the worst model, but it cannot be ruled out at the $3-\sigma$ level.

Assuming that the source is within M 81 and the distance to the galaxy is $3.6 \mathrm{Mpc}$ (Freeman et al. 1994), the black-body model implies an unabsorbed luminosity $\sim 6.7 \times 10^{38} \mathrm{erg} \mathrm{s}^{-1}$ in the $0.2-2.4 \mathrm{keV}$ band, and a negligible intrinsic absorption of the source in comparison with the line-of-sight Galactic absorption. If the flare spectrum is a power law then the luminosity in the ROSAT band is $\sim 8.5 \times 10^{38} \mathrm{erg} \mathrm{s}^{-1}$. Clearly if the power law extends to higher energies, then the X-ray luminosity could be significantly above $10^{39} \mathrm{erg} \mathrm{s}^{-1}$.

The Chandra data show that the source had a powerlaw spectrum in "quiescence". The best fit parameters are listed in Table 1 . The inferred unabsorbed luminosity is $1.7 \times 10^{37} \mathrm{erg} \mathrm{s}^{-1}$ in the $0.5-8 \mathrm{keV}$ band and, in the ROSAT bandpass, it is roughly 60 times fainter than that of the flare luminosity in late 1993. There is a suggestion that the Chandra spectrum is steeper than the ROSAT spectra; however we note that $\Gamma \sim 1.6$ is consistent with both data sets.

\section{Nature of the source}

The fact that the flaring spectra obtained by ROSAT PSPC in late 1993 are equally well fit by a number of simple models implies that the exact nature of the source is still open to investigation. The low absorbing column does tend to rule out a high mass companion, since such systems typically have strong stellar winds and hence large intrinsic columns. Here we briefly discuss several possibilities. We begin by assuming a single source is 


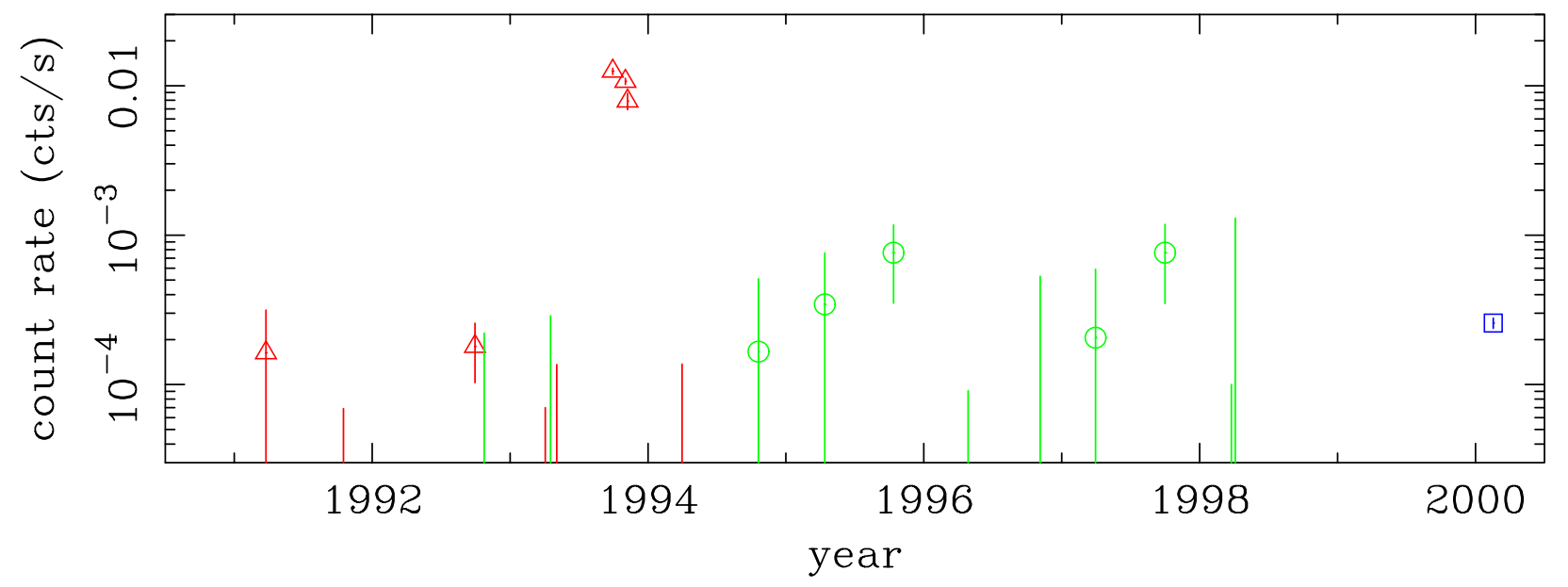

Fig. 3. The light curve of the transient between 1991 and 2000. The count rates are converted to equivalent ROSAT HRI count rates, assuming that the spectrum is a power law with $\Gamma=1.6$ and $n_{\mathrm{H}}=4.0 \times 10^{20} \mathrm{~cm}^{-2}$. Data before 2000 are the $R O S A T$ observations: triangles correspond to PSPC observations and circles correspond to HRI observations. The Chandra observation in 2000 May is represented by a square.

Table 1. Spectral fit to the ROSAT and Chandra observations.

\begin{tabular}{|c|c|c|c|c|c|c|}
\hline observation & date & model & $n_{\mathrm{H}}\left(10^{20} \mathrm{~cm}^{-2}\right)$ & $\Gamma$ & $T(\mathrm{keV})$ & $\chi^{2} /$ d.o.f. \\
\hline \multirow[t]{3}{*}{ ROSAT } & \multirow[t]{3}{*}{1993 Sep. 29} & phabs $*$ po & $4.4_{-1.8}^{+2.7}$ & \multirow[t]{3}{*}{$1.30_{-0.36}^{+0.37}$} & & $19.6 / 22$ \\
\hline & & $p h a b s * b b$ & 4.0 (fixed) & & $0.33_{-0.33}^{+0.05}$ & $31.6 / 23$ \\
\hline & & phabs $*$ brem & $4.3_{-1.2}^{+2.1}$ & & $15.5_{-10.1}^{+\infty}$ & $19.6 / 22$ \\
\hline \multirow[t]{3}{*}{ ROSAT } & \multirow[t]{3}{*}{1993 Nov. 1} & $p h a b s * p o$ & $6.3_{-3.2}^{+12.8}$ & \multirow[t]{3}{*}{$1.55_{-0.45}^{+0.95}$} & & $19.8 / 18$ \\
\hline & & $p h a b s * b b$ & 4.0 (fixed) & & $0.32_{-0.32}^{+0.05}$ & $22.5 / 19$ \\
\hline & & phabs $*$ brem & $5.6_{-2.4}^{+7.1}$ & & $4.7_{-2.3}^{+\infty}$ & $19.4 / 18$ \\
\hline \multirow[t]{3}{*}{ ROSAT } & \multirow[t]{3}{*}{ coadd Sep./Nov. } & $p h a b s * p o$ & $4.8_{-1.6}^{+2.6}$ & \multirow[t]{3}{*}{$1.37_{-0.27}^{+0.29}$} & & $46.0 / 43$ \\
\hline & & $p h a b s * b b$ & 4.0 (fixed) & & $0.32_{-0.02}^{+0.03}$ & $61.5 / 44$ \\
\hline & & phabs $*$ brem & $4.6_{-1.0}^{+1.9}$ & & $9.5_{-3.9}^{+\infty}$ & $45.6 / 43$ \\
\hline \multirow[t]{3}{*}{ Chandra } & \multirow[t]{3}{*}{2000 May 7} & phabs $*$ po & $12.4_{-12.4}^{+17.7}$ & \multirow[t]{3}{*}{$1.88_{-0.55}^{+0.60}$} & & $2.3 / 4$ \\
\hline & & $p h a b s * b b$ & 4.0 (fixed) & & $0.47_{-0.47}^{+0.10}$ & $10.6 / 5$ \\
\hline & & phabs $*$ brem & $7.6_{-7.6}^{+3.9}$ & & $4.5_{-0.9}^{+\infty}$ & $2.4 / 4$ \\
\hline
\end{tabular}

responsible for all the observed X-ray emission and describe possible scenarios for objects outside (Sect. 4.1) or within (Sect. 4.2) M 81. We then consider (Sect. 4.3) the possibility that two or more X-ray sources are present along the same line of sight.

\subsection{Systems outside $M 81$}

If we assume the X-rays come from a star within our Galaxy and that object ID 50777 in Perelmuter \& Racine (1995) is its counterpart, the colours suggest an early $K$ spectral type. If the star is on the main sequence, the optical brightness implies a distance of $1.5 \mathrm{kpc}$. We first consider the possibility that the X-rays are due to coronal emission. For stellar coronae, the ratio of the X-ray flux to the optical flux is expected to be in the range $10^{-3}$ to $10^{-5}$ for F- to M-type stars (Topka, et al. 1982) whereas we find $F_{\mathrm{x}} / F_{\text {opt }} \approx 0.14$. The hardness of the Chandra spectrum is also inconsistent with typical coronal emission. Therefore, coronal emission from a late-type main-sequence star is unlikely.

If a distance of $1.5 \mathrm{kpc}$ is assumed, then the flare luminosity of the source would be $\sim 10^{32} \mathrm{erg} \mathrm{s}^{-1}$. Typical X-ray luminosities (above $0.5 \mathrm{keV}$ ) of magnetic cataclysmic variables (mCVs) - which are close binaries consisting of a magnetic white dwarf and a late $\mathrm{K}$ or $\mathrm{M}$ main-sequence star - are about $10^{31}-10^{32} \mathrm{erg} \mathrm{s}^{-1}$ (see Cropper 1990 and references therein). MCVs are also known to show transitions between high and low states (Cropper 1990; Warner 1995). Thus we are unable to rule out a foreground $\mathrm{mCV}$ based on the X-ray observations alone. However, the optical source should not appear extended as it does in the Hubble image. The issue could be resolved if an optical image taken during the X-ray high state were available as mCVs show brightening in both the X-ray and optical bands during high states. 

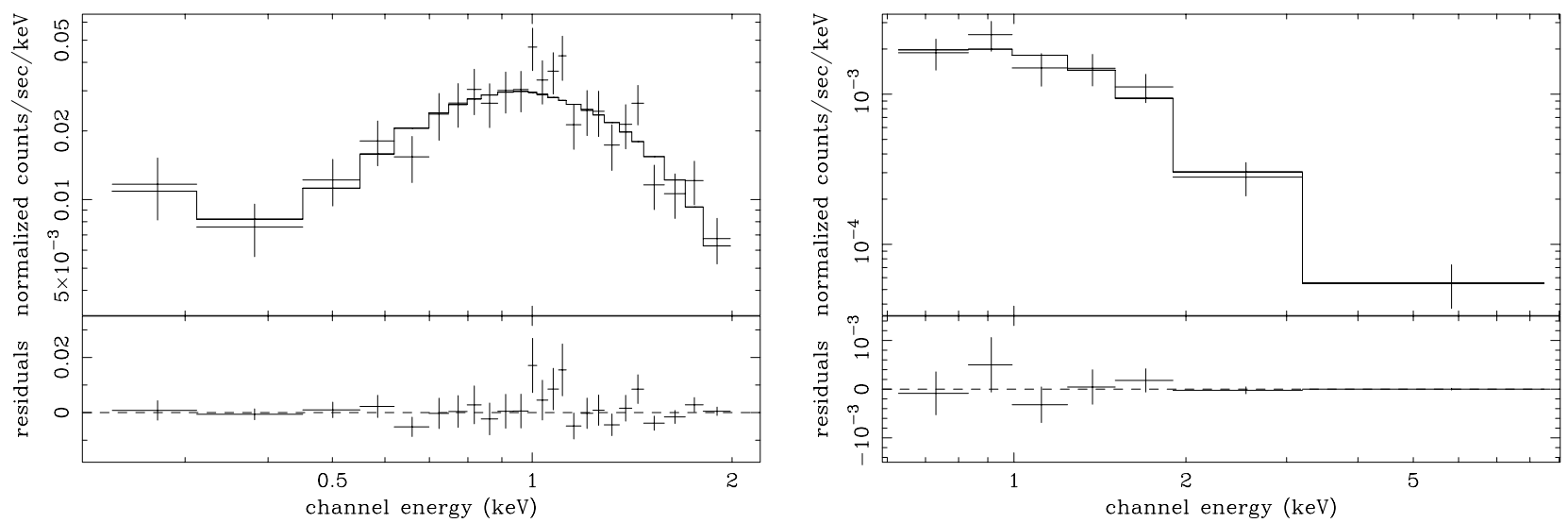

Fig. 4. Fits to the ROSAT and Chandra spectra of the source (left and right panel respectively). The $R O S A T$ spectrum was obtained on 1993 September 29 when the source was bright. The model of the fit is (phabs $*$ po) and the parameter values are listed in Table 1 . The source was relatively faint during the Chandra observation on 2000 May 7 . The spectrum is fit by a model (phabs * po) with the parameters listed in Table 1 .

The extended emission could be accounted for if the object were a background AGN. AGNs are not known to show such large amplitude X-ray variabiltiy on timescales of a few months as seen. In principle tidal disruption of a star by the black hole at the center of a galaxy could produce a large amplitude $\left(10^{3}\right.$ to $\left.10^{4}\right)$ flare but the expected timescale is of order a year or more (Ulmer 1999). Furthermore, if the object is behind M 81, then the absorbing column would come from not only our own galaxy, but also the column through the entire disk of M 81 as well as any intrinsic column. The low column measured from the X-ray data makes this possibility unlikely. An optical measurement of spectral redshift would provide conclusive evidence.

If the optical source is unrelated to the X-ray source, then the true optical counterpart would be a fainter and presumably more distant object. The most common X-ray source is an interacting binary. X-ray binaries in their active states have luminosities in the range $10^{36}-10^{38} \mathrm{erg} \mathrm{s}^{-1}$ (e.g. White et al. 1995). But, the ROSAT count rate during the flare in 1993 implies a luminosity of only $\sim 5 \times 10^{35} \mathrm{erg} \mathrm{s}^{-1}$ in the $0.2-2.4 \mathrm{keV}$ band even at a distance as great as $100 \mathrm{kpc}$, which would place it in the outer part of the Galactic halo. If the source is an X-ray binary within the Galaxy, unrelated to the observed optical source, it would be one of the fainter systems.

\subsection{Systems within $M 81$}

If we assume that the optical object is in M 81 and is related to the X-ray source, then it is far too bright to be an isolated X-ray binary in M 81. It is also too bright to be an isolated supernova remnant which could potentially account for the optical extension, but not the X-ray variability.

The optical object may be a previously unidentified (and yet to be confirmed) globular cluster in M 81 harbouring the X-ray source. Galaxies similar to M 81 could contain of order 200 globular clusters (Perelmuter \& Racine 1995). To date, only 25 globular clusters in M 81 have been confirmed (Perelmuter et al. 1995), and only 4 of these are within the viewing field of ACIS S3. The colours reported by Perelmuter \& Racine (1995) for ID 50777 are within their expectations for M 81 globular cluster candidates. As shown in Sect. 2, object ID 50777 has a half light radius of about 0.2 arcsec which would be impossible to detect from the ground, although the outer halo could be. The $V$ magnitude of 16.9 would imply an absolute magnitude of $M_{V} \approx-10.9$ which would make it brighter than any Milky Way cluster and similar to the absolute magnitude of the brightest globular cluster in the Andromeda galaxy M 31 (see the review by Ashman \& Zepf 1998). Thus the brightness and small size of ID 50777 would account for it being missed in previous searches for globular clusters.

X-ray transients are known to occur in Milky Way globulars. Typical globular cluster sources in our galaxy have luminosities ranging up to $\sim 5 \times 10^{37} \mathrm{erg} \mathrm{s}^{-1}$ and none are black hole candidates. The luminosity of the Chandra source is consistent with that of bright X-ray sources found in Galactic globular clusters (Verbunt \& van den Heuvel 1995; Sidoli et al. 2001). Both the spectra of the ROSAT observations in late 1993 and the Chandra observation are well fit by a power-law with a photon index about 1.6. This value is consistent with the power-law component seen in the spectra of X-ray binaries. The ROSAT count rates together with the distance to M 81 imply the flare luminosity of the source above the Eddington luminosity of X-ray binaries containing an accreting neutron star. Di Stefano et al. (2001) report Chandra observations of M 31 that show that the majority of the most luminous X-ray sources in M 31 are in globular clusters and that the highest sustained luminosity observed exceeds $2 \times 10^{38} \mathrm{erg} \mathrm{s}^{-1}$. We also note that some peculiar Galactic neutron-star binaries (e.g. Cir X-1, which is believed to have a very eccentric orbit) have super-Eddington luminosities (up to three times the Eddington limit of a 1.5- $M_{\odot}$ accretor) over a quite substantial portion of their orbital cycles (e.g., Shirey et al. 1996). Thus the possibility of an extremely bright neutronstar X-ray binary cannot be easily eliminated. 
The high luminosity makes the source a candidate black-hole binary. From the Chandra observation we obtain a "quiescence" luminosity of $1.7 \times 10^{37} \mathrm{erg} \mathrm{s}^{-1}$. The ratio of the flare luminosity to the quiescence luminosity is therefore $\sim 60$. This is significantly lower than those of the Galactic black-hole binaries GS 2000+251, Nova Mus 91, A0620-00, and V404 Cyg whose observed ratios $\left(10^{6}\right.$ to $10^{7}$, Garcia et al. 2001) are in agreement with Advection-Dominated-Accretion-Flow (ADAF) model predictions (Narayan et al. 1997).

\subsection{Multiple $X$-ray sources in a M 81 globular cluster}

Although the X-ray temporal behaviour suggests an X-ray transient, the peak luminosity is difficult to explain with a neutron star. On the other hand the brightness of Chandra measurement is inconsistent with the currently popular ADAF model. This leads to the possibility that the strong source seen by ROSAT in 1993 and the fainter source observed by Chandra in 2000 are two different objects. If we assume that each detected X-ray source occupies an area of the detector out to the $90 \%$ radius of the Chandra PSF then the 97 X-ray sources reported in Tennant et al. (2001) would occupy $\sim 2 \%$ of all the pixels on the ACIS S3 detector. Thus the probability of a randomly-located source appearing within a detected ACIS source region is $2 \%$. Of course, assuming X-ray sources are more likely to be found in dense stellar environments, such as the cores of globular clusters, then this probability should be considered a lower limit.

Thus, object ID 50777 may be a massive (i.e., luminous and compact) globular cluster in M 81 containing the ROSAT flare source P33 and the fainter Chandra source. The Chandra source is presumably one or more persistent X-ray binaries while the brightness of the ROSAT source in 1993 suggests a black-hole binary in the core.

If systems displaying similar X-ray characteristics can be identified elsewhere, however, the multiple-source hypothesis becomes less viable and a single-source explanation is to be favored. We note that the Einstein source X-2 lies very close to a bright $(V=17.29)$ point-like object listed as ID 50548 in Perelmuter \& Racine (1995). During the Chandra observation, X-2 had faded dramatically relative to its Einstein-measured brightness. To our knowledge, however, X-2 has never exceeded an X-ray luminosity of $\sim 2 \times 10^{38} \mathrm{erg} \mathrm{s}^{-1}$. Optical followups of ID 50548 would also be of interest. The recently discovered transient source (Steinle et al. 2000) in the direction of galaxy Cen A may also display characteristics similar to those reported here. If the source is in Cen A, then it would have had a peak X-ray luminosity of $3 \times 10^{39} \mathrm{erg} \mathrm{s}^{-1}$ and it also appears to be associated with a weak Chandra source.

Roughly $10 \%$ of globular clusters are expected to contain accreting neutron stars detectable above the Chandra threshold and galaxies like M 81 typically contain of order 200 globular clusters (Perelmuter \& Racine 1995). However, there are only 25 confirmed globular clusters in M 81 (Perelmuter et al. 1995) and only 10 within the Chandra-observed field. None of these are coincident with X-ray sources detected by Chandra.

\section{Summary}

The transient X-ray source brightened to $\sim 7 \times 10^{38} \mathrm{erg} \mathrm{s}^{-1}$, during ROSAT observations spanning 1993 Sep. 29 to 1993 Nov. 7 and was only $12-75$ times fainter during the rest of the 10-year observing period including observations taken 5 months before and after the bright phase. Spectra obtained during both the high and low brightness states can be equally well fit by either power law models with $\Gamma \sim 1.6$ and by thermal bremsstrahlung models with temperatures exceeding $4 \mathrm{keV}$. In all cases the data are consistent with only a Galactic interstellar absorption column. Blackbody models provide a substantially poorer fit. The bright optical counterpart to the transient source is extended with a half-light radius of $\sim 0.2$ arcsec and with luminosity and colours consistent with a massive globular cluster in M 81 .

The only viable foreground object candidate for this source is a $\mathrm{mCV}$. A Galactic $\mathrm{mCV}$ would have a flaring luminosity (at a distance $\sim 1.5 \mathrm{kpc}$ ) and spectral shape similar to that observed. The optical extension and low state X-ray luminosity of order $\sim 2 \times 10^{30} \mathrm{erg} \mathrm{s}^{-1}$ argue strongly against this hypothesis. A background AGN is unlikely based on the short X-ray "on-time" of the source and on the low column density derived from the spectral fits.

An X-ray binary at the distance of M 81 is suggested by the shape of the observed power law spectra yet the luminosities observed make this scenario difficult to reconcile with canonical Galactic objects in this class: the high luminosity during the flare exceeds that of an accreting neutron star while the small ratio of flare luminosity to quiescent luminosity argues against a black-hole system. This can be resolved if the X-rays come from two objects, a black hole that produced the flare and a neutron star to account for the Chandra flux. Though a rare superposition may occur, the probability is greater if the sources are located within a single massive globular cluster in M 81. If this is the case, then this is the first black hole candidate in a globular cluster. In any case, the peak X-ray luminosity exceeds that of any globular cluster X-ray source in the Galaxy and rivals that of the brightest M 31 globular cluster X-ray source.

Further optical observations are encouraged in order to confirm the nature of the optical counterpart and to monitor changes in optical brightness if the source turns out to be a foreground object. An optical redshift measurement would strictly limit the AGN hypothesis. The companion star of a foreground $\mathrm{mCV}$ should show distinct emission lines unlike the absorption line spectrum typical of globular clusters.

Acknowledgements. K. W. thanks Martin Weisskopf for funding his visits to MSFC. Support for this research was provided in part by NASA/Chandra grant GO0-1058X. 


\section{References}

Arnaud, K. 1996, in Astronomical Data Analysis Software and Systems V., ASP Conf. Ser., 101, 17

Ashman, K. M., \& Zepf, S. E. 1998, Globular Cluster Systems (Cambridge University Press, Cambridge)

Bloser, P. F., Grindlay, J. E., Kaaret, P., et al. 2000, ApJ, 542, 1000

Cropper, M. S. 1990, Sp. Sci. Rev., 54, 195

Di Stefano, R., Kong, A. K. H., Garcia, M. R., et al. 2001 [astro-ph/0106254]

Fabbiano, G. 1988, ApJ, 325, 544

Freeman, W. L., et al. 1994, ApJ, 427, 628

Garcia, M. R., McClintock, J. E., Narayan, R., et al. 2001, ApJ, 553, L47

Immler, S., \& Wang, Q. D. 2001 [astro-ph/0102021]

Krist, J., \& Hook, R. 1999, The Tiny Tim User's Guide http://www.stsci.edu/software/tinytim

La Parola, V., Peres, G., Fabbiano, G., Kim, D. W., \& Bocchino, F. 2001 [astro-ph/0103250]

Lewin, W. H. G., van Paradijs, J., \& van den Heuvel, E. P. J., 1995, X-ray Binaries (Cambridge University Press, Cambridge)

Ma, C., Arias, E. F., Eubanks, T. M., et al. 1998, AJ, 116, 516

Narayan, R., Garcia, M. R., \& McClintock, J. E. 1997, ApJ, 478, L79
Perelmuter, J.-M., Brodie, J. P., \& Huchra, J. P. 1995, AJ, 110, 620

Perelmuter, J.-M., \& Racine, R. 1995, AJ, 109, 1055

Shirey, R. E., Bradt, H. V., Levine, A. M., \& Morgan, E. H. 1996, ApJ, 469, 21

Sidoli, L., Parmar, A. N., Oosterbroek, T., et al. 2001, A\&A, 368,451

Stark, A. A., Gammies, C. F., Wilson, R. W., et al. 1992, ApJS, 79,77

Steinle, H., Dennerl, K., \& Englhauser, J. 2000, A\&A, 357, L57

Swartz, D. A., et al. 2001, in preparation

Tennant, A. F., Wu, K., Ghosh, K. K., Kolodziejczak, J. J., \& Swartz, D. A. 2001, ApJ, 549, L43

Topka, K, Avni, Y., Golub, L., et al. 1982, ApJ, 259, 677

Ulmer, A. 1999, ApJ, 514, 180

Verbunt, F., \& van den Heuvel, E. P. J. 1995, in X-ray Binaries, ed. W. H. G. Lewin, J. van Paradijs, \& E. P. J. van den Heuvel (Cambridge University Press, Cambridge), 457

Warner, B. 1995, Cataclysmic Variable Stars (Cambridge University Press, Cambridge)

White, N. E., Nagase, F. \& Parmar, A. N. 1995, in X-ray Binaries, ed. W. H. G. Lewin, J. van Paradijs, \& E. P. J. van den Heuvel (Cambridge University Press, Cambridge) 